\title{
Control de un sistema de descargas de barrera dieléctrica basado en el diseño gráfico de
}

\section{sistemas}

\section{Control of a Dielectric Barrier Discharge Reactor based on Graphical System Design}

\section{Jiménez-Aviles Gerardo}

Universidad Autónoma del Estado de México

Facultad de Química

Departamento de Posgrado

Correo:gerjimav@gmail.com

\section{Valdivia-Barrientos Ricardo}

Instituto Nacional de Investigaciones Nucleares

Departamento de Estudios del Ambiente

Laboratorio de Aplicaciones de Plasmas

Correo: ricardo.valdivia@inin.gob.mx

\section{Colín-Cruz Arturo}

Universidad Autónoma del Estado de México

Facultad de Química

Departamento de Posgrado

Correo: acolinc@uaemex.mx

\author{
Pacheco-Pacheco Marquidia \\ Instituto Nacional de Investigaciones Nucleares \\ Departamento de Estudios del Ambiente \\ Laboratorio de Aplicaciones de Plasmas \\ Correo: marquidia.pacheco@inin.gob.mx \\ Pacheco-Sotelo Joel \\ Instituto Nacional de Investigaciones Nucleares \\ Departamento de Estudios del Ambiente \\ Laboratorio de Aplicaciones de Plasmas \\ Correo: joel.pacheco@inin.gob.mx
}

\section{Resumen}

Este trabajo describe el desarrollo e implementación de un sistema de monitoreo y control de los parámetros físicos involucrados en las etapas de inicio, sostenimiento y apagado de descargas de barrera dieléctrica generadas dentro de un reactor de geometría coaxial. En el proceso se empleó el diseño gráfico de sistemas (GSD, graphical system design) para conjuntar eficientemente una interfaz gráfica de usuario (GUI, graphical user interface), la tarjeta de adquisición de datos DAQ-6259 con canal de comunicación USB, una fuente de voltaje conmutado push-pull, controladores de flujo gaseoso y equipo de diagnóstico químico y electrónico. El núcleo del enfoque GSD se encuentra en el código de programación G de LabVIEW ${ }^{\mathrm{TM}}$; de esta manera, se recopilaron datos precisos y en tiempo real de las variables (vgr.: flujo de aire y potencia aplicada), mismos que permitieron realizar un análisis del desempeño de las descargas.

Durante las pruebas se emplearon diferentes concentraciones de una mezcla de helio contaminada con óxidos de nitrógeno, con la finalidad de cuantificar el porcentaje de degradación/destrucción de los últimos. Los resultados indican un valor mayor a $88 \%$. Por seguridad, a la GUI se accede a través de la intranet institucional, debido a que los compuestos generados pueden representar un riesgo para la salud humana.

Descriptores: contaminación atmosférica, descargas de barrera dieléctrica, diseño gráfico de sistemas, fuentes móviles, óxidos de nitrógeno, plasma frío.

\begin{abstract}
This paper describes the development and implementation of a control and monitoring system for the physical parameters involved in the stages of initiation, maintenance and shutdown of dielectric barrier discharges generated within a coaxial geometry reactor. In the process, the graphical system design (GSD) was employed to efficiently combine a graphical user interface (GUI), the data acquisition card DAQ-6259 with USB communication channel, a switch-mode power supply in push-pull configuration, drivers for gas flow, chemical and electronic equipment for diagnosis. The core of the GSD approach is located in the G code from LabVIEW TM, in this way, accurate data was collected in real time (e.g., airflow and power applied), which allowed to perform an analysis of the discharges performance. During testing, different concentrations of a mixture of helium contaminated with nitrogen oxides, in order to quantify the percentage of its degradation. The results indicate a value greater than $88 \%$. For safety reasons, the GUI can be accessed remotely through institutional intranet, because of the generated compounds that may represent a risk to human health.
\end{abstract}

Keywords: atmospheric pollution, dielectric barrier discharges, graphical system design, mobile sources, nitrogen oxides, cold plasma. 
INTRODUCCIÓN

Sólido-líquido-gas es una secuencia conocida de los estados de agregación de la materia al considerar aumentos graduales de la energía cinética de las moléculas que la componen. Desde la rigidez y orden de un sólido se puede conseguir que sus uniones se rompan totamente y que cada molécula se mueva libremente hasta formar un gas, medio que generalmente tiene carga co. Para producir conductividad eléctrica en un gas se cuministra energía suficiente para disociar sus mó́celas en los átomos que las componen y, así, extraer a los electrones de sus estados fundamentales hacia niveles superiores, generando un flujo de partículs cargadas cuyo comportamiento dinámico se domina por fuadas electromagnéticas (Conrads, 2000). Esta materia ionizada es plasma, comúnmente obtenida por compresió adiabática, al incidir un haz de fotones o por colisiones atómicas, a escala industrial se utiliza energía eléctrica por ser económica y técnicamente más viable (Becker 2010).

El estudio del plasma suele dividirse de acuerdo con el equilibrio termico de las particulas que lo componen: plasmas térmico y frío. En esta última clasificacón se encuentran las descargas corona, luminiscentes y de barrera dieléctrica (DBD) (Conrads, 2000; Becker, 2010). Los plasmas fuera de equilibrio termodinámico local, no termicos o frios (NTP, non-thermal plasma) se caracterizan por la alta divergencia en las energías de sus electrones libres y especies pesadas (átomos, iones $\left(T_{+}\right)$, partículas neutras $\left(T_{n}\right)$ y moleculas). Generalmente, la temperatura de las particulas pesadas permanece entre 300 y $400 \mathrm{~K}$, mientras que la de los electrones $\left(T_{d}\right)$

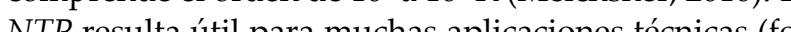
cos, televisores, tratamiento de superficies, fabricacó de nanomateriales, la ingenieŕ́ la nanolitografía) porque es posible controlar separada mente a $T$ y $T$ de $T$ Las características que diferencian mente a $T_{+} \mathrm{y} T$
al NTP son:

1) Su alta desviación cinética de equilibrio: $T_{e} \approx 10^{4} \mathrm{~K} \gg T_{+}>T$

2) Su baja densidad electrónica, de $10^{14}$ a $10^{18} \mathrm{~m}^{-3} ; \mathrm{y}$ 3) La temperatura promedio $\left(T_{g}=T_{+}+T_{n}\right)$ se mantiene similar a la del ambiente, debido a que la masa de
las especies pesadas es mucho mayor que la de lo electrones, de aquí el nombre de NTP (Becker, 2010).

Son pocos los avances en el campo del NTP que se han traducido con éxito del laboratorio a tecnologías aplica- bles en la vida cotidiana; impedidos, entre otros factores, por la falta de identificación, cuantificación control de los subproductos producidos en el proceso, demás de que a altas presiones es difícil mantener la descarga debido a su inestabilidad y a susceptibilidad a a filamentación, seguida de una transición a arco eléctico (Kunhardt, 2000). La aplicación ambiental histórca e ideal de las DBD se enfoca a la generación de ozono (gas oxidante), pues necestla funcionar a temperatura baja porque las presión relativamente alta (Gottschalko, requiere una prismo modo, sistemas a (Gerie NTP-catalizar 2010 . Del combinado para tratar óxidos de nitrógeno (NO) provenientes de automóviles: comprenden una etapa pridativa (NTP) que convierte monóxido de nitrógeno (NO) a dióxido de nitrógeno $\left(\mathrm{NO}_{2}\right)$, y una fase de reducción y almacenamiento (catalizador), que convierte el $\mathrm{NO}_{2}$ a nitrógeno $\left(\mathrm{N}_{2}\right)$, dióxido de carbono $\left(\mathrm{CO}_{2}\right)$, hidrógeno $\left(\mathrm{H}_{2}\right)$ y oxígeno $(\mathrm{O})$ (Penetrante et al., 2002). Dado que las DBD operan a niveles altos de potencia y tratan flujos grandes de gas a presión atmosférica con caída de presión insignificante (basado en la tecnología madura de $\mathrm{O}_{3}$ ), potenciales aplicaciones en el control de la contaminacion atmosferica se han examinado sisteGSD, de un reactor para DBD aplicado al tratamiento de $\mathrm{NO}$

\section{DESCARGAS DE BARRERA DIELÉCTRICA}

En el NTP la $T_{e}$ es el factor determinante al iniciar las reacciones químicas, que en técnicas activadas térmicamente resultarian ineficientes o imposibles de realizar. Úa la ejecutan los electrones $\left(e^{-}\right)$más lige nenegía la ejecutan los electrones (e), más ligeros que da, por lo que pueden acelerarse eficazmente para repartir la energía al resto del gas mediante colisiones dentro de las cules se encuentran: las éásticas, que cambian la energía cinética de las especies neutras, qu inelásticas, que convierten a las especies neutras en especies reactivas e inducen una serie de reacciones quí$\begin{array}{lll}\text { micas (tabla } & 1 \text { ) que producen simultáneamente }\end{array}$ portadores de carga y especies altamente reactivas en el NTP (Meichsner, 2010). En (tabla 1) $X_{2}$ representa las moléculas diatómicas, $X$ átomos, $X_{2}^{+}$moléculas diatómicas que ceden electrón, $X_{2}^{-}$moléculas diatómicas que ganan electrón, $X^{-}$átomo que gana electrón, $X^{+}$átomo que cede electrơn $X_{2}$ y moléculas diatómicas excitada. conjunto de canales de conducción (microdescargas) en el espacio existente entre dos electrodos, separados por una o varias capas dieléctricas (vidrio, slilice, polimeros o cerámicos), sobre los cuales se aplica voltaje alterno para niciar y sostener las microdescargas. Las geometrías tipicas en los reactores son: de placas paralelas (planar coplanar) o cilíndricas (coaxial) (Kogelschatz, 2003).

Pacheco et al. (2008; 2012; 2013) reportan investigaciones teórico-experimentales de tratamiento para gases de combustión mediante DBD y se puntualiza e culos). En su trabajo de 2008 fuentes móviles (vehicinética química obtuvieron el mecanismo de degrada cón de los $\mathrm{NO}_{\mathrm{y}} \mathrm{y}$ determinaron el comportamento de cos ron coaxiales, de una y dos paredes de pírex con brecha de $1.4 \mathrm{~cm}$ y volumen para descarga de $65 \mathrm{Hm}^{3}$. $\mathrm{En}$ l investigación de Estrada et al. (2011) con un consumo de $20 \mathrm{~W}$, se trató la mezcla de He (gas de acarreo), aire, vapor de agua y $\mathrm{NO}_{x}$ en un reactor de pírex, logrand obtener valores de remoción de $99.74 \%$ y $96.66 \%$ para niveles de concentración inicial de $\mathrm{NO}_{x}$ de 50 y 300 ppm, respectivamente.

Tabla 1. Reacciones en la Región Activa del NTP Ionización $\quad \mathrm{X}_{2}+e^{-} \rightarrow \mathrm{X}_{2}^{+}+2 e^{-}$ Captura de electrones

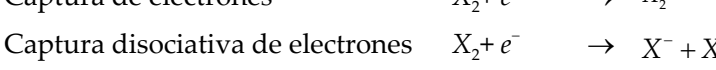
Disociación $\quad X_{2}+e^{-} \rightarrow X+X+e^{-}$

Excitación

Ionización disociativa $\mathrm{X}_{2}+e^{-} \rightarrow \mathrm{X}_{2}^{9}+e^{-}$

Datos extraídos de Meichsner (2010)

En las diversas aplicaciones de las DBD es importante conocer su comportamiento para poder cuantificar $y$ nalizar los diversos fenómenos que ocurren durante el roceso por acción de las variables involucradas (flujo voltaje aplicado o corriente demandada). Existen dive sos dispositivos para realizar esta tarea, que van desde sensores individuales hasta controladores automatizados muy sofisticados (Jiménez et al., 2012).

DISEÑ Gráfico de SISTEMAS

El GSD es una técnica de diseño (embebido), prototipo prueba, control y despliegue de aplicaciones, que combina programación gráfica en paralelo con hardware COTS (commercial off-the-shelf) para obtener la mayor cantidad de calculo util con un costo comercial bajo simplificar el desarrollo de una aplicacion, dando como resultado diseños robustos personalizados. El GSD per- mite diseñar sin la necesidad de hacer uso del hardware final, posibilitando la realización de simulaciones acortando los ciclos de integración e implementación (Bishop, 2007). En este proceso de instrumentación se emplearon:

1) LabVIEW 2013 para programar una GUI de un instrumento virtual (VI, virtual instrument);

La tarjeta de adquisición de datos reconfigurable NI

La GUI centraliza el monitoreo y control de los dispositivos asociados a las DBD, mientras recopila datos precisos en tiempo real.

\section{MONTAE DEL EXPERIMENTO}

Esencialmente, para generar DBD se emplea un flujo gaseoso, una fuente de voltaje alterno y un reactor que confine las microdescargas. En el proyecto se empleó un reactor coaxial $\left(\mathrm{C}-\mathrm{DBD}_{2}\right)$ elaborado de alúmina, el suministro de gas se llevó a cabo a traves de dos válvulas de control y un mezclador. Como fuente de excitación se diseño y construyo un sistema de potencia integrado por una fuente de voltaje de $C D$ y un inversor de alto voltaje a alta frecuencia, caracterizado con osciloscopios (AEMC® OX 7104 III y GWO Instek GDS-122), sondas de alto voltaje (Tektronix $\circledast$ P6015A) y corriente (Fluke® 80i-1000s). El porcentaje de degradación del reactor se calculó con los datos proporcionados por el analizador de gases residuales (RGA, residual gas analyzer) Horiba○ PG-250. Una vez instalado el experimento, se iniciaron las pruebas con diferentes concentraciones en la mezcla $73.97 \mathrm{kPa}$ y temperatura promedio de $294 \mathrm{~K}$.

LíNEAS DE GAS

A partir de los estudios realizados por Pacheco $e t a l$. (2012), que establecen la composición y concentraciones de una muestra sintética de gas de combustión, en este trabajo se usaron dos gases adquiridos en INFRA®: la mezcla CAM 96 Middle Range (CAM), compuesta de propano $\left(\mathrm{C}_{3} \mathrm{H}_{8}\right)$, monóxido de carbono $(\mathrm{CO}), \mathrm{CO}_{2}$ y NO, nistrar la mezcla gaseosa se instalaron dos válvulas controladoras de flujo másico (MFC, mass flow controller), una MKS ${ }^{\mathrm{TM}}$ MFC-M1000B $\left(M F C_{1}\right)$ para la CAM, y una Omega ${ }^{\mathrm{TM}} \mathrm{FMA}-\mathrm{A} 2315\left(\mathrm{MFC}_{2}\right)$ para el gas de acarreo, en este caso el He; ambas valvulas fueron conectadas a un controlador MKS ${ }^{\mathrm{TM}}$ 247D. Debido a que los 
$M F C$ se encuentran calibrados para flujos de $\mathrm{N}_{2^{\prime}}$, eajustaron las escalas para el gas correspondiente con ayud de las Ecuaciones 1-4 (MKS, 2013a; MKS, 2013b; Ome$\mathrm{ga}$, 2013). Los ajustes realizados se agrupan en la tabla 2 , donde al sumar los valores de la última columna se determina que el flujo máximo de la mezcla He-CAM es de $67.751 \mathrm{~min}^{-1}$, en una relación porcentual 85.84 $14.16 \%$

$G C F_{C A M}=0.3106 \sum_{i=1}^{n} a_{i} s_{i}\left(\sum_{i=1}^{n} a_{i} d_{i} C p_{i}\right)^{-1}$

$S C F=(G F)\left(G C F_{C A M}\right)$

Flujo $_{C A M}=\left(G C F_{C A M}\right)\left(F l u j_{N_{N 2}}\right)$

Flujo $_{\mathrm{He}}=1.454$ Flujo $_{\mathrm{N} 2}$

donde

$G C F_{C A M}=$ factor de corrección de la mezcla CAM compuesta de $n$ gases,

densidad del i-ésimo gas

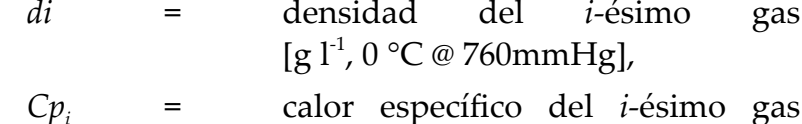

$\begin{aligned} C p_{i}= & \text { calor espect } \\ & {\left[\text { cal g g }{ }^{\circ} \mathrm{C}^{-1}\right] }\end{aligned}$

$a_{i}=$ fracción de flujo del $i$-ésimo gas,

$s_{i} \quad=\quad$ factor de corrección debido a la estructura molecular del $i$-ésimo gas. igual a:

1.030 para gases monoatómicos,

1.000 para gases diatómicos,

0.941 para gases triatómicos,

0.880 para gases poliatómicos,

SCF = factor de corrección de escala,

$G F \quad=\quad$ factor de corrección de la válvula

FUeNTE DE VOLTAJE

Para generar y mantener las DBD es necesario alimentar continuamente el sistema con una fuente bipolar, en caso contrario, el medio retorna a la fase gaseosa. La amplitud mínima de voltaje para que sucedan las descargas se denomina Voltaje de Rompimiento $\left(V_{V}\right)$, cuando su valor es inferior al requerido la ionización es prácticamente nula una vez superado ligeramente ese nivel comienza a existir un flujo de corriente entre los electrodos (plasma parcialmente ionizado). El valor de $V_{r}$ depende del gas, la presión de trabajo (P) y de la distancia entre los electroos (d), parámetros vinculados con la Fórmula de Paschen (Ecuación 5, en la que $\delta$ y $\sigma$ son constantes específicas del gas inyectado) (Fridman, 2008).

$$
V_{r}(p d)=\frac{\delta p d}{\sigma+\ln (p d)}
$$

Si al C-DBD 2 se acopla una fuente de voltaje alterno de alta frecuencia, tanto la eficiencia como la conductivirad eléctrica aumentan y el tamaño del equipo final se de excitación radica en su influencia en el componcmiento general de la descarga donde en el orden de $\mathrm{kHz}$ los electrones y los iones siguen las oscilaciones del campo eléctrico, mientras que en un orden superior, $\mathrm{MHz}$ o $\mathrm{GHz}$, solo los electrones siguen dicho campo (Roth, 1995). Una fuente conmutada (SMPS, switch-mode power supply) permite trabajar con frecuencias de $\mathrm{kHz}$ mediante el cambio de estado de transistores. En este trabajo se ocupa como fuente principal de voltaje $\left(\mathrm{V}_{\mathrm{PP}}\right)$ la SMPS B\&K@ PRECISION XLN10014, que puede proporcionar una potencia máxima de $1.44 \mathrm{~kW}(100$ $\mathrm{V} @ 14.4$ A), el diseño del sistema de excitación se enfoca en desarrollar un inversor con topología push-pul (PPI, push-pull inverter) con base en la teoria expues por Bersani (2009) y Mohammad (2007) (figura 1).

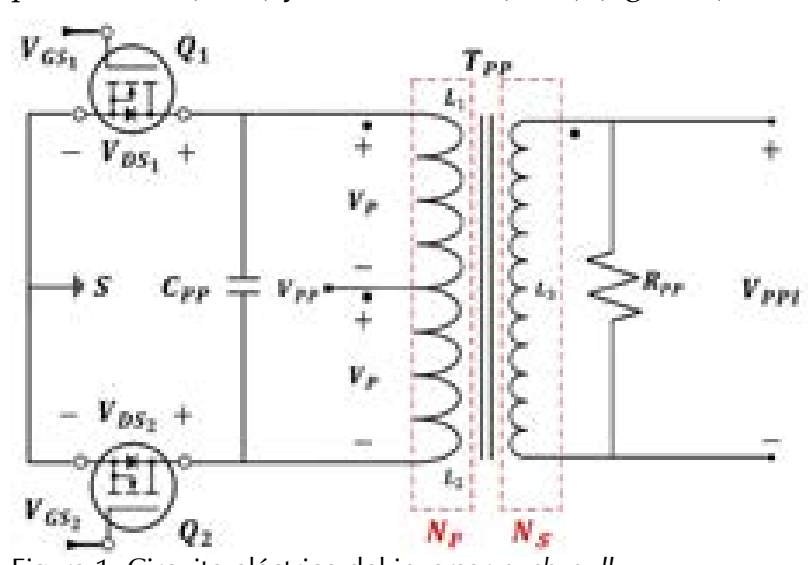

Figura 1. Circuito eléctrico del inversor push-pull

En el diagrama eléctrico del PPI se ubican los MOSFET $Q_{1}$ y $Q_{2}$, matrícula IXFN80N50 $\left(V_{\text {DSMÁX) }}=500 \mathrm{~V}\right.$, $\mathrm{I}_{\mathrm{DS}(\mathrm{MÁX})}=80 \mathrm{~A}$ y $\left.\mathrm{R}_{\mathrm{DS}(\mathrm{MÁX})}=55 \mathrm{~m} \Omega\right)$, $\mathrm{C}_{\mathrm{pP}}$ es un condensado de poliéster de $3.2 \mathrm{nF}$ y forma el circuito taque resonante con el transformador $\mathrm{T}_{\mathrm{Py}}$ compuesto por las bobinas $\mathrm{L}_{1}, \mathrm{~L}_{2}$ (ambas de $\left.320 \mu \mathrm{H}\right)$ y $\mathrm{L}_{3}(5 \mathrm{mH})$, con una ganancia $M$ determinada experimentalmente de 100 cada vez que se activa uno de los devanados primarios. El núcleo
de $T_{\mathrm{PP}}\left(\mathrm{U}_{\mathrm{pP}}\right)$ se compone de ferrita grado 3C94, Ferrox-

\begin{tabular}{|c|c|c|c|c|c|c|c|}
\hline Gas & $\begin{array}{r}d_{i} \\
{\left[\mathrm{~g} \mathrm{I}^{-1}\right]}\end{array}$ & $\begin{array}{c}C P_{i} \\
{\left[{ }_{[c a l ~}^{\left.g^{-1}{ }^{-1} C^{-1}\right]}\right.}\end{array}$ & $\begin{array}{c}\text { GCF } \\
\text { individual }\end{array}$ & $\begin{array}{c}\text { Concentración } \\
\text { [\%vol.] }\end{array}$ & $\begin{array}{c}\text { Error } \\
{[\%]}\end{array}$ & $\begin{array}{l}\text { Factor de } \\
\text { Corrección }\end{array}$ & $\begin{array}{c}\mathrm{MFC} \\
{\left[1 \mathrm{~min}^{-1}\right]}\end{array}$ \\
\hline Helio & 0.1786 & 1.2410 & - & 100 & - & 1.4540 & 58.15 \\
\hline CAM & & & & & & 0.9594 & 9.59 \\
\hline $\mathrm{C}_{3} \mathrm{H}_{8}$ & 1.9670 & 0.3885 & 0.36 & 0.119988 & \pm 1.0 & - & - \\
\hline $\mathrm{CO}$ & 1.2500 & 0.2488 & 1.00 & 3.99 & \pm 1.0 & - & - \\
\hline $\mathrm{CO}_{2}$ & 1.9640 & 0.2016 & 0.70 & 11.99 & \pm 1.0 & - & - \\
\hline NO & 1.3390 & 0.2328 & 0.99 & 0.30006 & \pm 1.0 & - & - \\
\hline $\mathrm{N}_{2}$ & 1.2500 & 0.2485 & 1.00 & 83.599952 & \pm 4.0 & - & - \\
\hline
\end{tabular}

cube@ U126/91/20-3C94 ( $\mathrm{L}_{\mathrm{UPP}}=48 \mathrm{~cm}, \mathrm{~A}_{\mathrm{UPP}}=5.6 \mathrm{~cm}^{2}$ $\mu \mathrm{PP}=2050$, con un peso de $1.36 \mathrm{~kg}$ ) (Ferroxcube, 2013). En paralelo a $L_{3}$ se conectó una resistencia $R_{\mathrm{pp}}$ de $100 \mathrm{M} @ 10 \mathrm{~W}$ para proporcionar una vía de descarga a la corriente en el devanado secundario una vez que se han extinguido las microdescargas. Con estos valores, aplicar la Ecuación 6

$$
f_{R} \frac{1}{2 \pi \sqrt{C_{P P} L_{P P}}}
$$

REACTOR

De acuerdo con la figura 2, las dimensiones y características físicas del C-DBD ${ }_{2}$ son: $15.40 \mathrm{~mm}$ de radio exterior $\left(R_{1}\right), 12 \mathrm{~mm}$ de radio interno $\left(R_{2}\right), 19.30 \mathrm{~cm}$ de longitud $\left(D_{1}\right), 4.85 \mathrm{~mm}$ de radio del aislante interno ( $\left(\mathrm{B}_{3}\right.$ ) el voltae de salida de PPI $\left(\mathrm{V}_{\text {pro }}\right)$ se conecta a $\mathrm{HV}$ que es un tubo hueco de acero inoxidable de $13.62 \mathrm{~cm}$ de longitud, 1.57 $\mathrm{mm}$ de grosor y radio de $3.16 \mathrm{~mm}\left(\mathrm{R}_{4}\right)$; el electrodo GND es una malla de acero inoxidable de $0.80 \mathrm{~mm}$ de grosor y $11.37 \mathrm{~cm}$ de longitud $\left(\mathrm{D}_{2}\right)$. El volumen de las microdescargas es de $50.60 \mu \mathrm{m}^{3}$. El reactor se elabor con dos capas aislantes de alumina, ya que propician

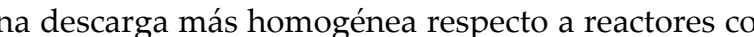
una barrera.

TARIETA DE ADQUIIICIÓN DE DATOS

La tarjeta 6259 cuenta con 128 conectores de entrada/saida en tiempo real, que incluyen 36 puertos analógicos: 4 salidas de 16 bits $(\mathrm{AO}, \pm 10 \mathrm{~V}) @ 2.00 \mathrm{MS} \mathrm{s}^{-1}, \mathrm{y} 32$ entradas $(\mathrm{AI}, \pm 10 \mathrm{~V}) @ 1.00 \mathrm{MS} \mathrm{s}^{2}$, que pueden referenciarse

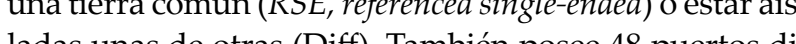

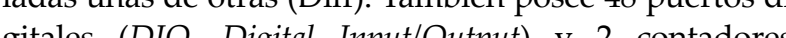

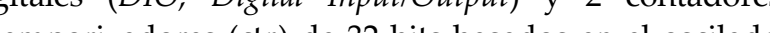
interno de $80 \mathrm{MHz}$ (National Instruments, 2013).

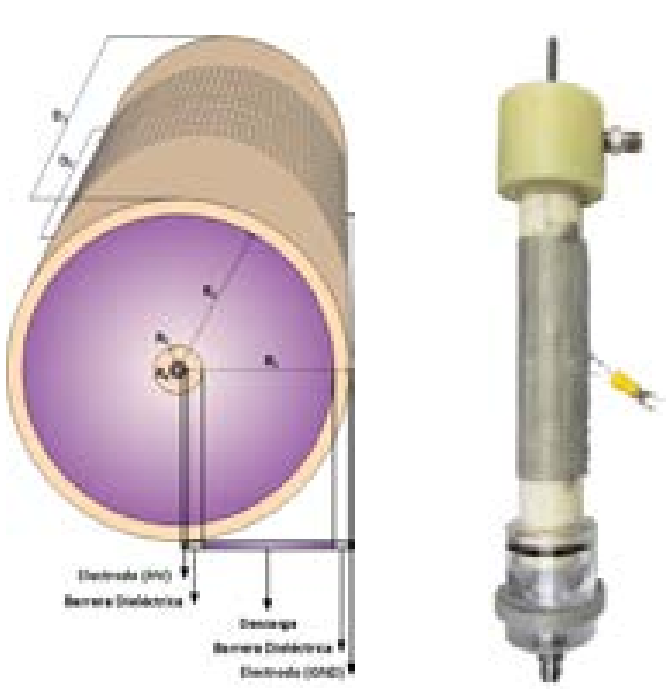

Figura 2. Reactor coaxial de doble barrera dieléctrica

Para acceder al control remoto de las válvulas de gadel controlador 247D, desde su panel frontal se ajustan las palancas de nivel de flujo y de modo de control a las posiciones Rem y Ext, respectivamente. Ext permite el control de la señal de flujo desde un dispositivo externo y Rem especifica que el cambio de estado del MFC será con lógica inversa TTL (TTL, Transistor-Transistor Lo$g i c$. Cada uno de los MFC requiere de dos señales de control: un pulso TTL DIO y un voltaje entre $0-5 \mathrm{~V}$ que es linealmente proporcional al flujo (AO). Por el mismo pás $\mathrm{DB}_{25}$ se envian estas dos señales y se recibe una

Para controlar el $P P I$, en la tarjeta se configura un para el control por Modulación de Ancho de Pulso (PWM pulse zidth modulation): $140 \mathrm{~Hz} \leq f, 160 \mathrm{kHz}$ y $0.01 \leq D_{\text {pwM }} \leq 0.99$, para la frecuencia y el ciclo de trabajo, respectivamente. La señal de se envía por los pines 10 
(señal) y 11 (tierra en serie con $R_{8}=390 \Omega$ ) al optoacoplador TLP250, cuya salida sirve como reloj para el flip-flop 7476 , que tiene sus entradas $J K=5 \mathrm{~V}$; este arreglo proporciona los pulsos $Q$ y $Q$ destasados $180^{\circ}$ entre sí, a los que se les aplica la funcion AND con la señal $c t r 0$ para obtener pulsos, $V_{G S 1}$ y $V_{G S 2}$ con rangos de la mitad de $c t r 0$ $H z \leq f_{P W M} \leq 80 \mathrm{kHz}$ y $0.005 \leq D_{P W M} \leq 0.495$ (figura 3).

Para la etapa de caracterización, se integraron los datos del $R G A$ PG-250 que entrega mediciones para $7 \mathrm{ga}-$ ses, sin embrago, solo se presentan los resultados para las ajustes que vande 0 a 5000 ppmy de 0 a $25 \%$.

\section{INTERTAZ GRÁEICA DE USUARIO}

El VI final se compone por cuatro sub-VI que permiten

a) Acceder al personal (Registro.vi)

b) Configurar los puertos de la USB-6259 y procesa los datos (Principal.vi)

c) Comunicar con el equipo de diagnóstico (PG250.vi) d) Guardar información (Bitácora.vi)

La información y los controles se pueden consultar modificar in situ o se puede ingresar remotamente a través de la dirección http://200.15.118.244/KaVi_v.2.04. html; simultáneamente, la rutina puede guardar infor-
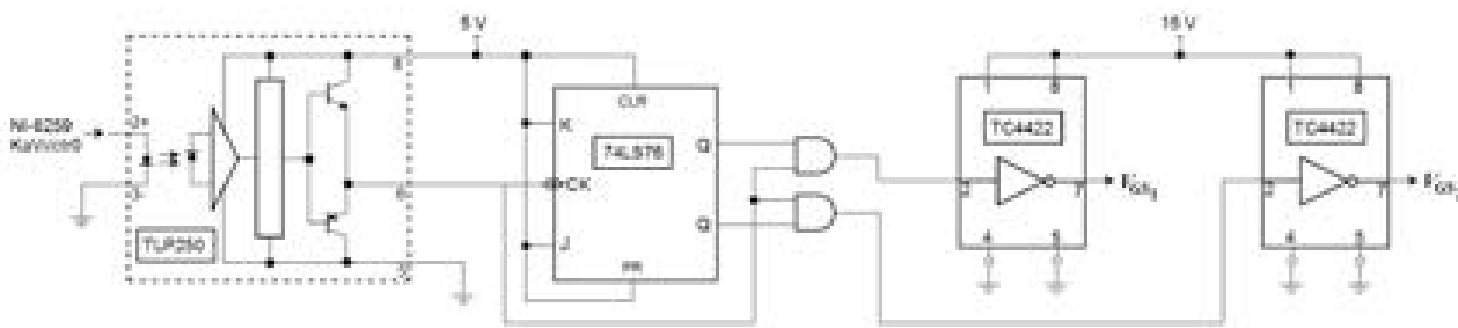

Figura 3. Circuito de control de compuerta para los MOSFET

Tabla 3. Distribución de los Puertos de la Tarjeta USB-6259

\begin{tabular}{|c|c|c|c|c|c|c|c|}
\hline \multicolumn{2}{|c|}{ Canal analógico de salida } & \multicolumn{2}{|c|}{ Canal analógico de entrada } & \multicolumn{2}{|c|}{ Canal digital } & \multicolumn{2}{|c|}{$\begin{array}{c}\text { Contador } \\
\text { temporizador }\end{array}$} \\
\hline Puerto & Dispositivo & Puerto & Dispositivo & Puerto & Dispositivo & Puerto & PWM \\
\hline \multirow{8}{*}{$\begin{array}{l}\mathrm{AO}_{0} \\
\mathrm{AO}_{1}\end{array}$} & $\mathrm{MFC}_{1}$ & $\mathrm{AI}_{0}-\mathrm{AI}_{8}$ & $\mathrm{MFC}_{1}$ & $\mathrm{DIO}_{0}$ & $\mathrm{MFC}_{1}$ & $\mathrm{ctr}_{0}$ & $\mathrm{Q}_{1}$ \\
\hline & & $\mathrm{AI}_{1}-\mathrm{AI} \mathrm{I}_{2}$ & $\mathrm{MFC}_{2}$ & & & & \\
\hline & & $\mathrm{AI}_{2}-\mathrm{AI}_{10}$ & $\mathrm{RGANO}_{x}$ & & & & \\
\hline & & $\mathrm{AI}_{3}-\mathrm{AI}_{11}$ & & & & & \\
\hline & & $\mathrm{AI}_{4}-\mathrm{AI}_{12}$ & & & & & \\
\hline & & $\mathrm{AI}_{5}-\mathrm{AI}_{13}$ & RGA & & & & \\
\hline & & $\mathrm{AI}_{6}-\mathrm{AI}_{14}$ & & & & & \\
\hline & & $\mathrm{AI}_{16}-\mathrm{AI}_{24}$ & & & & & \\
\hline
\end{tabular}
misma que apaga los dispositivos. dicadores.
6. Esquema. Sobrepone un diagrama con los voltajes y las corrientes en puntos estratégicos. Los valore provienen de lecturas del osciloscopio, de la fuente de voltaje, de los sensores y son calculados.

. XLN. Estos tres controles interactúan vía USB con la fuente XLN10014. Posibilitan habilitar/inhibir la salida de potencia, mientras establecen limites de corriente y voltaje. Cuenta con indicadores que muestran los valores reales de voltaje, corriente y potencia suministrados al reactor.

1. Operador. Al inicio una ventana pide complementar los campos Nombre y Clave de usuario, son autentificados con una base de datos para autorizar el uso del equipo, el programa se detiene al no coincidir.

2. Encendido. Una vez pasado el control de acceso, este indicador se muestra de color verde y comienza te configuración de los puertos de la USB-6259. Para terminar la prueba se debe presionar y un cuadro
emergente pide la confirmación de la instrucción

3. Logotipo. Al presionarlo despliega una ventana con

información de la institución colaboradora.

4. Ayuda. Activa/desactiva las etiquetas de ayuda mostradas al mover el cursor sobre los controles/in-

Congelar. Mantiene estática la pantalla de control para evitar su modificación por error.

Push Pull. Permite modificar los parámetros del

modificar las concentraciones y el

flujo de gas. riables, por intervalos de segundo minuto u hora. Al fnalizar la prueba comprime la carpeta, la envía por correo electrónico y elimina el archivo *.zip creado. 11. RGA. Abre la interfaz de control y adquisición de PG-250.

Con estas herramientas instaladas se creó un sistema basado en el enfoque GSD y se prosiguió con una serie experimental, en la que se preparo una mezcla de CAM diluida con He en concentraciones que parten del 0.38 al $13.95 \%$ vol., aplicando voltajes desde la fuente principal de 25 a $55 \mathrm{~V}$ (tabla 4 )

En la figura 5 se presenta una fotografía y su descripción del montaje del sistema desarrollado en el laboratorio de aplicaciones de plasmas del Instituto Nacional de Investigaciones Nucleares. En la figura $6 \mathrm{se}$

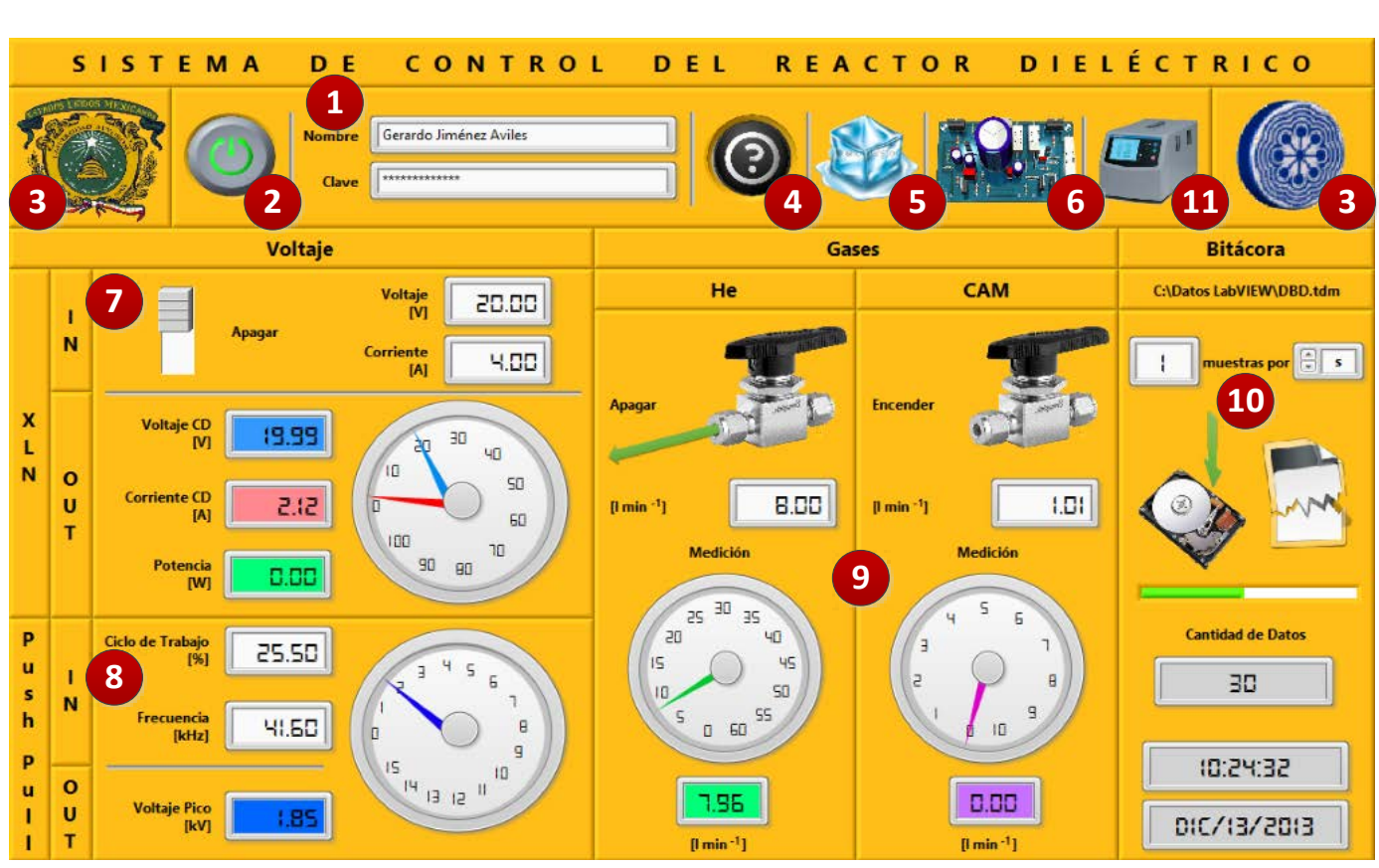

Figura 4. Panel principal de la interfaz gráfica de usuario para control del reactor C-DBD presenta un diagrama de interconexión de todo el sistema, es decir, la interfaz gráfica de usuario, la tarjeta de adquisición de datos, la fuente de voltaje conmutada, los controladores de flujo, el inversor de alto voltaje y el reactor coaxial de barrera dieléctric

$$
\text { Resultados }
$$

Después de realizar un barrido de frecuencias, se encontró que la transferencia máxima de potencia a las microdescargas se logra con trenes de pulsos de $12 \mathrm{~V}$ de tos dos parámetrz a $25.5 \%$ de servicio, si se varing versor será mayor, sin embargo, las descargas serán inestables y la fase entre la señales de voltaje y corriente incrementa (factor de potencia). Las gráficas de la figura 8 representan este caso, entre más se alejan los valores $f_{P W M}$ y $D_{P W M}$ de sus ideales es mayor el voltaje de salida, pero también el desfasamiento (figuras $7 \mathrm{~b}$ y $7 \mathrm{c}$ ). En las gráficas se marcó la corriente de resonancia, que corresponde a los procesos de ionización y recombinación. Además, el voltaje visto por los transistores se eleva hasta 4.5 veces el suministrado (figura 8), cuando idealmente debería ser de 2.6 veces. La potencia de entrada se obtuvo al multiplicar los valores de la corriente y el voltaje obtenidos con la interfaz virtual, mientras que la poPoia de salida se calculó mediante la Fórmula de (1943) a través de un algoritmo en MathCADo, con la razón de ambas potencias, se obtuvo que el índice de eficiencia del siste- 


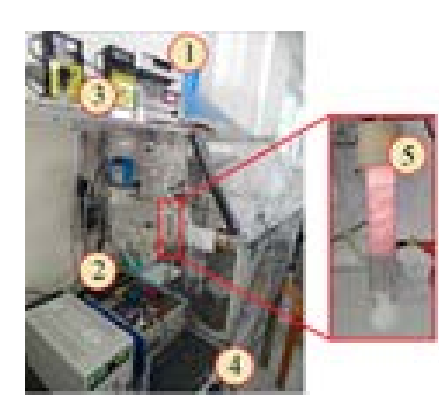

\section{Tajecta de adquisicioin de datos USB-6259}

Comeroladors de flujo $M F C 1$ y?

4. Fuentec conmutada XLN10014

S. Reacher DED encendido

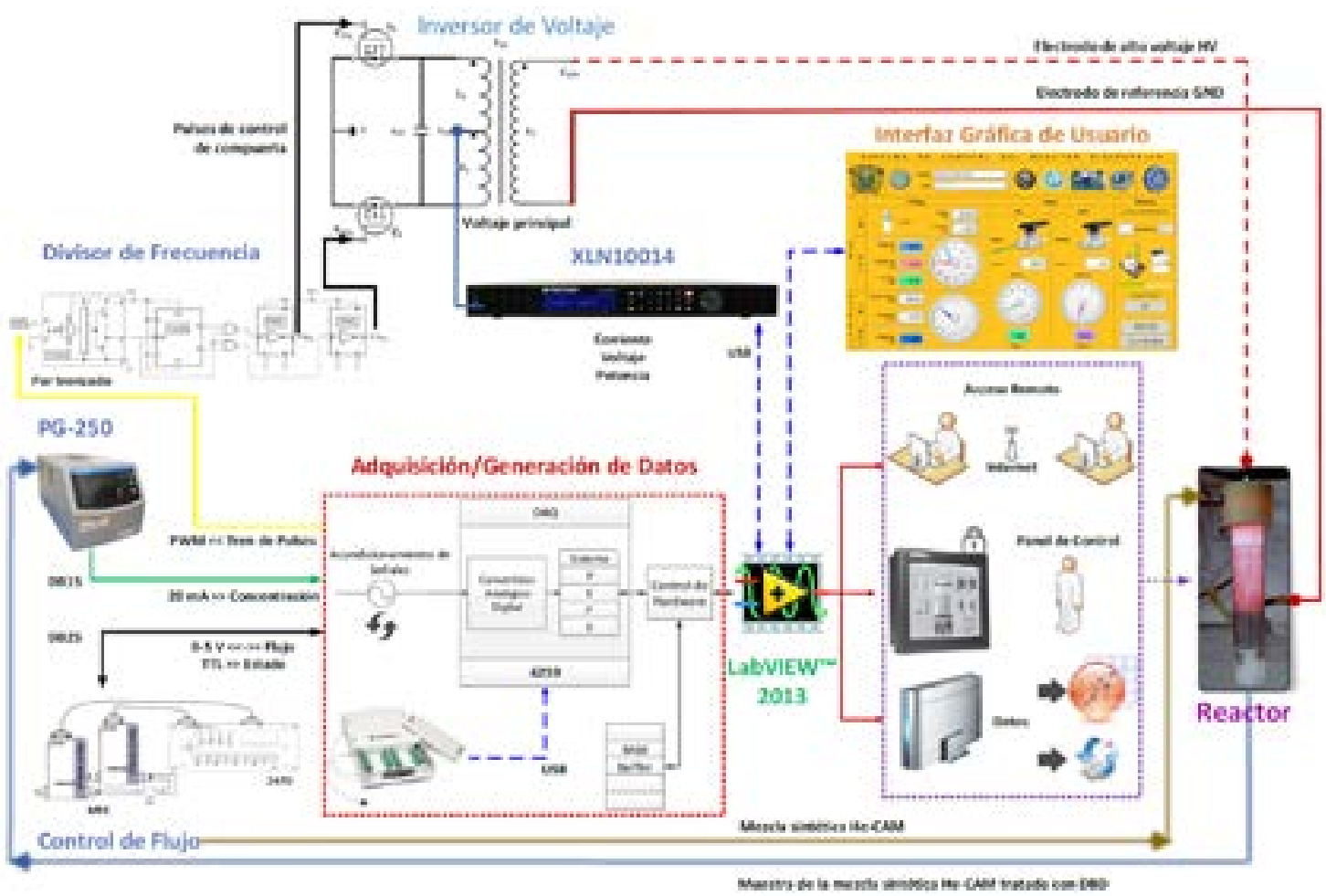

Figura 6. Diagrama de interconexión de todo el sistema

ma de potencia es en promedio del 0.90 , mismo que varía al filamentarse las descargas.

Las condiciones mínimas para que inicien las descargas son: $\mathrm{He}=1.236 \mathrm{lmin}^{-1}, \mathrm{~V}_{\mathrm{Pp}}=19 \mathrm{~V}, \mathrm{~V}_{\mathrm{PP}(\mathrm{k})}=1.88 \mathrm{kV}$ 3.7 W; con ello, el sistema puede trabajar en estado estacionario con un flujo reducido de $0.15 \mathrm{~min}^{-1}$ de He y un consumo de 32 W. En la figura 9 se observan las formas de onda del voltaje aplicado, la corriente y la potencia demandadas; al incrementar gradualmente el flujo decae la corriente y las descargas se extinguen (zona de standby). Posteriormente se hizo fluir una mezcla de He-CAM a las concentraciones y flujos enlistados en la tabla 4 con potenciales de 25 a $55 \mathrm{~V}$; cada una de las concentraciones para la fila de $55 \mathrm{~V}$ corresponde a los tiempos $t_{x}$ identificados en la figura 10, también se entregan los valores de la densidad volumétrica de potencia (DVP: potencia distribuida por unidad de volumen disponible trada (DEE: cantidad de energía aplicada por volumen de gas). Con ambos conceptos, DVP y DEE, se construye el concepto Valor Fuente que permite visualizar las cantidades de potencia y energía necesarias por unidad de volumen $\left(\mu \mathrm{m}^{3} \mathrm{y}\right.$ l) para cada dato de degradació obtenido.

$\mathrm{Al}$ aplicar $25 \mathrm{~V}$ y hacer fluir He se identifican oscilaciones subitas de $0-t_{1}$ en la gráfica de la potencia (debdas a la corriente, ya que el voltaje se mantien constante), suavizadas inmediatamente al incluir CAM en $0.38 \%$ vol; en este primer caso se consideraron las muestras de 0.38 y $1.14 \%$ vol. de CAM, que trabajaron en estado estacionario con un consumo promedio de 7 W. Posteriormente se aumentó la concentración de CAM y la potencia decayó instantaneamente con la consecuente extinción total de las microdescargas. Para el caso de $35 \mathrm{~V}$, se repiten las concentraciones anteriores y se nota un ascenso de la potencia sin que esto reflej n aumento proporcional de degradación.

Ll procedimiento se repite para 45 y $55 \mathrm{~V}$. En la figura de $t_{1}-t_{2}$ la rampa de potencia aumenta $8 \mathrm{~W}$ y la debidos a la ene

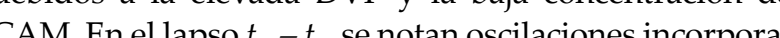
das en la gráfica derivadas de la filomentación homogé-

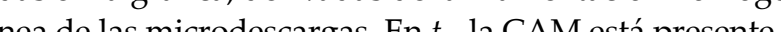
$13.95 \%$ vol y se eleva a $15.61 \%$ vol el sistema permanece por un instante hasta que la potencia decae naturalmente en $t_{12}$. Más allá de esta última concentración las DBD tienden a localizarse dentro del reactor y a extinguirse. El valor máximo de degradación de $\mathrm{NO}_{x}$ es de $88.24 \%$ para una concentración inicial (CI) del $0.38 \%$ (9 ppm), en un flujo total de $12.481 \mathrm{~min}^{-1} \mathrm{y}$ con $V_{P P}=25 \mathrm{~V}$ (DVP=141.50 mW $\mu^{-3} @ D E E=34.42 \mathrm{~J}^{-1}$ ); este específico no refleja una degradación mayor para $V_{p p}=35$ 45V, a pesar de que la DVP y la DEE se intensifican 2
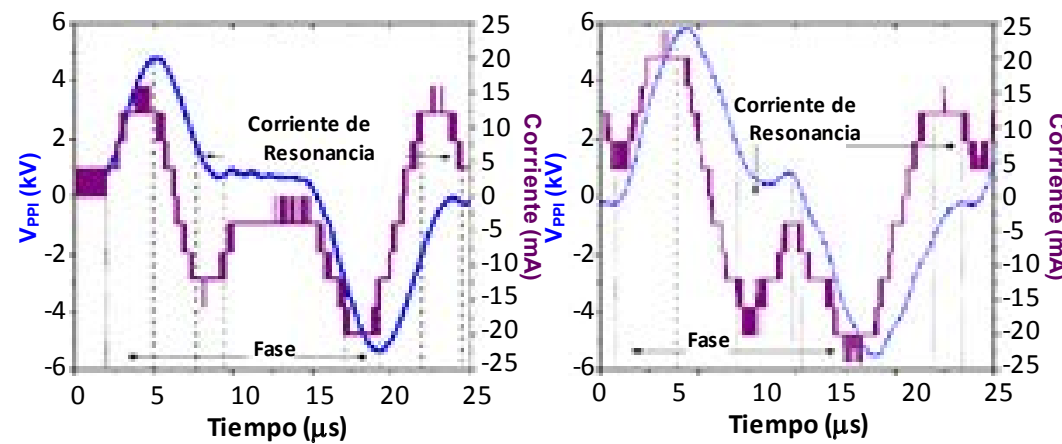

determ

$\begin{array}{cc}10 & 15 \\ \text { Tiempo } & (\mu s)\end{array}$

4 veces su valor inicial, respectivamente. Se identifica que el porcentaje de degradación aumenta en cada experimento para la misma muestra conforme se incrementa $V_{P P}$ para flujos mayores a $0.141 \mathrm{~min}^{-1}$ de CAM (1.14 \%vol., 24 ppm de $\mathrm{NO}_{\mathrm{x}}$ ); en estos casos sí es congruente el aumento de la DVP y la DEE. El valor maximo es de $74.07 \%$ de degradación cuando $V_{P P}=55 \mathrm{~V}$; sin embargo, cuando el flujo de CAM es máxino (2.02 1 Con lo an

ies de pruebas y CONCLUSIONES

Se diseñó y elaboró un sistema de instrumentación y control robustos, basado en el GSD para equipos de plasma frío tipo descargas de barrera dieléctrica para su aplicacion en el tratamiento de gases de combustión en el que se incluyó el acondicionamiento de líneas de suministro de gases, la programación de una interfaz gráfica de usuario, y un inversor con topología pushpull de alto voltaje y alta frecuencia.

Se identificaron las condiciones idoneas del circuito de control de potencia a $41.3 \mathrm{kHz}$ con $25.5 \%$ de ciclo de trabajo, parámetros que permitieron generar una señal

Figura 7. Variación de $f_{P W M}$ y $D_{P W M}$ para dete
$D_{P W M}=25.5 \%$, c) $f_{P W M}=56 \mathrm{kHz}$ y $D_{P W M}=20 \%$

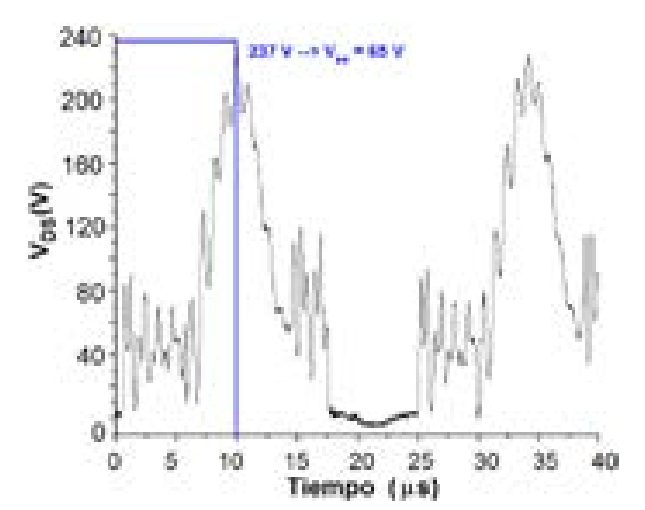

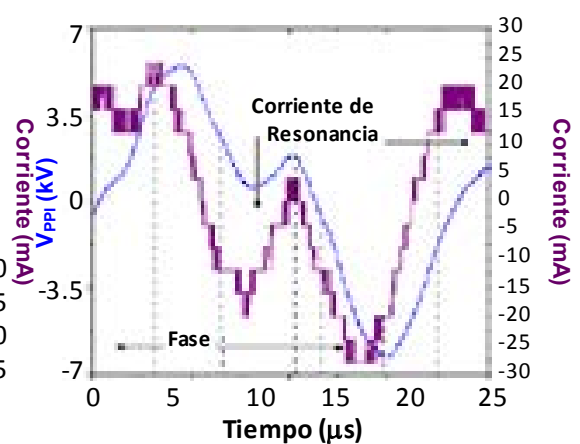

Figura 8. Detección de valores pico para estimar el voltaje
máximo de operación del MOSFET

IST 
senoidal modificada en la etapa de potencia. Fuera de estos parámetros la descarga se vuele inestable; la modificación del $D_{P W M}$ provoca un cambio directo en la amplitud de salida, mientras que la frecuencia en la capacitancia es equivalente del reactor.

Se realizo el estudio de degradación de $\mathrm{NO}_{x}$ diluido en la mezcla sintética CAM 96 Middle Range y helio industrial. Las corridas experimentales arrojaron porcentajes de degradacion superion a $88 \%$ para los $\mathrm{NO}^{-}$ En la bibiografla se obtuvieron resultados de degradataje se debe a que en esta investigación se inchye la jerch ${ }^{2}$

\section{Tabla 4. Datos de degradación de $\mathrm{NO}_{\mathrm{x}}$ diluido en la mezcla CAM-He}

\begin{tabular}{|c|c|c|c|c|c|c|}
\hline $\begin{array}{l}V_{p p} \\
\text { [V] }\end{array}$ & $\begin{array}{c}\text { DVP } \\
{\left[\mathrm{mW} \mu \mathrm{m}^{-3}\right]}\end{array}$ & $\begin{array}{l}\text { DEE } \\
{\left[\mathrm{J}^{-1}\right]}\end{array}$ & $\begin{array}{c}\text { Flujo Total } \\
{\left[1 \mathrm{~min}^{-1}\right]}\end{array}$ & $\begin{array}{l}\text { CAM } \\
\text { [vol.] }\end{array}$ & $\begin{array}{l}\mathrm{CINO}_{\mathrm{x}} \\
{[\mathrm{ppm}]}\end{array}$ & $\begin{array}{c}\text { Degradación } \\
{[\%]}\end{array}$ \\
\hline \multirow[t]{2}{*}{25} & 141.50 & 34.42 & 12.48 & 0.38 & 9.00 & 88.24 \\
\hline & 130.83 & 31.57 & 12.58 & 1.14 & 24.00 & 40.43 \\
\hline \multirow[t]{6}{*}{35} & 290.91 & 70.77 & 12.48 & 0.38 & 9.00 & 61.11 \\
\hline & 279.64 & 67.49 & 12.58 & 1.14 & 24.00 & 55.10 \\
\hline & 273.12 & 65.45 & 12.67 & 1.89 & 39.00 & 41.03 \\
\hline & 273.32 & 65.24 & 12.72 & 2.26 & 47.50 & 37.89 \\
\hline & 273.72 & 64.82 & 12.82 & 2.99 & 62.50 & 34.40 \\
\hline & 272.13 & 64.00 & 12.91 & 3.72 & 80.00 & 33.13 \\
\hline \multirow[t]{9}{*}{45} & 583.79 & 142.02 & 12.48 & 0.38 & 9.00 & 70.00 \\
\hline & 583.79 & 140.90 & 12.58 & 1.14 & 24.00 & 64.00 \\
\hline & 570.35 & 136.67 & 12.67 & 1.89 & 39.00 & 54.32 \\
\hline & 558.50 & 133.30 & 12.72 & 2.26 & 47.50 & 48.96 \\
\hline & 538.14 & 127.44 & 12.82 & 2.99 & 62.50 & 42.97 \\
\hline & 522.33 & 122.84 & 12.91 & 3.72 & 80.00 & 39.51 \\
\hline & 503.56 & 116.70 & 13.10 & 5.13 & 115.00 & 35.37 \\
\hline & 492.89 & 112.51 & 13.30 & 6.49 & 148.00 & 31.42 \\
\hline & 460.67 & 103.68 & 13.49 & 7.82 & 185.00 & 23.89 \\
\hline \multirow[t]{12}{*}{55} & 996.64 & 240.52 & 12.58 & 1.14 & 24.00 & 74.07 \\
\hline & 994.07 & 238.20 & 12.67 & 1.89 & 39.00 & 67.82 \\
\hline & 986.56 & 235.47 & 12.72 & 2.26 & 47.50 & 64.08 \\
\hline & 980.83 & 232.28 & 12.82 & 2.99 & 62.50 & 60.29 \\
\hline & 967.39 & 227.50 & 12.91 & 3.72 & 80.00 & 56.47 \\
\hline & 904.55 & 209.63 & 13.10 & 5.13 & 115.00 & 49.79 \\
\hline & 843.87 & 192.63 & 13.30 & 6.49 & 148.00 & 44.81 \\
\hline & 801.19 & 180.31 & 13.49 & 7.82 & 185.00 & 38.52 \\
\hline & 775.69 & 172.15 & 13.68 & 9.12 & 219.00 & 33.26 \\
\hline & 752.37 & 164.69 & 13.87 & 10.38 & 248.00 & 28.97 \\
\hline & 731.23 & 156.78 & 14.16 & 12.20 & 287.00 & 24.35 \\
\hline & 661.46 & 138.98 & 14.45 & 13.95 & 330.00 & 19.27 \\
\hline
\end{tabular}

del gas $y$, por ende, la descarga tiende a filamentarse y arcar un volumen menor dentro del reactor, mientras que en los trabajos reportados únicamente se incluye $\mathrm{NO}_{x}$ y al gas de acarreo (He o Ar).

La tendencia clara en el tratamiento de los $\mathrm{NO}_{x}$ se encamina a un descenso en la capacidad del sistema su degradación respecto al aumento en la concentración de CAM, acompanada de altos niveles de DVP DEE para bajas concentraciones, mismos que dismin yen confor de $\mathrm{NO}$ - Debe a CAM a la mezcla hasta las 30

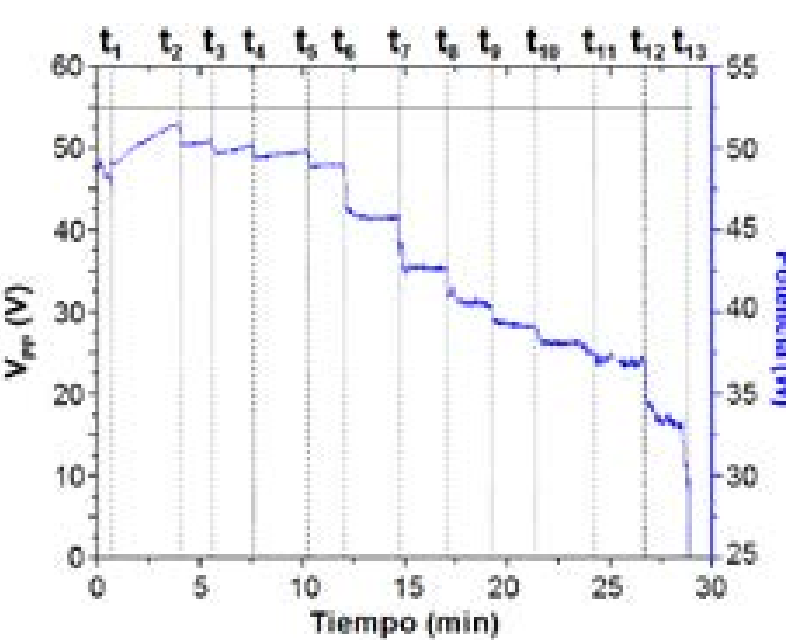

Figura 10. Voltajes máximo (55 V) y potencia aplicados al

su total entendimiento y empleo posterior en el tratamiento a bordo de gases de escape.

La integración fácil de tecnologías nuevas a la plaforma GSD permite que en futuras mejoras se empleen módulos similares a la serie MQ para sensa gases y un microcontrolador que sustituya a la 6259 con la consecuente reducción de costos a una fración del precio actual.

\section{Agradecimientos}

Los autores agradecen a Alfonso Salazar, Fidel Ramos Gustavo Soria, Mario Ibañez, Miguel Durán, Migue Hidalgo y Moisés Garcia por su soporte técnico en e Laboratorio de Aplicaciones de Plasmas, al Instituto Nacional de Investigaciones Nucleares por las facilidades y recursos proporcionados para la realización de CVU/Becatio: 412287/258202).

\section{REFERENCIAS}

cer KH. tion to Complex Plasmas, Berlin, Springer 2010, pp. 367-394. tion to Complex Plasmas, Berlin, Springer 2010, pp. 367-394. Microchip Technology Inc, 2009, ID; AN1207.

Bishop R H. Mechatronic system control, logic, and data acouisition The Mechatronics Handbook, 2a ed., CRC Press, 2007, ISBN 9781420009026 .

Conrads H. y Schmidt M. Plasma generation and plasma sources. Plasma Sources Science and Technology, volumen 9 (número 4) 2000: 441.

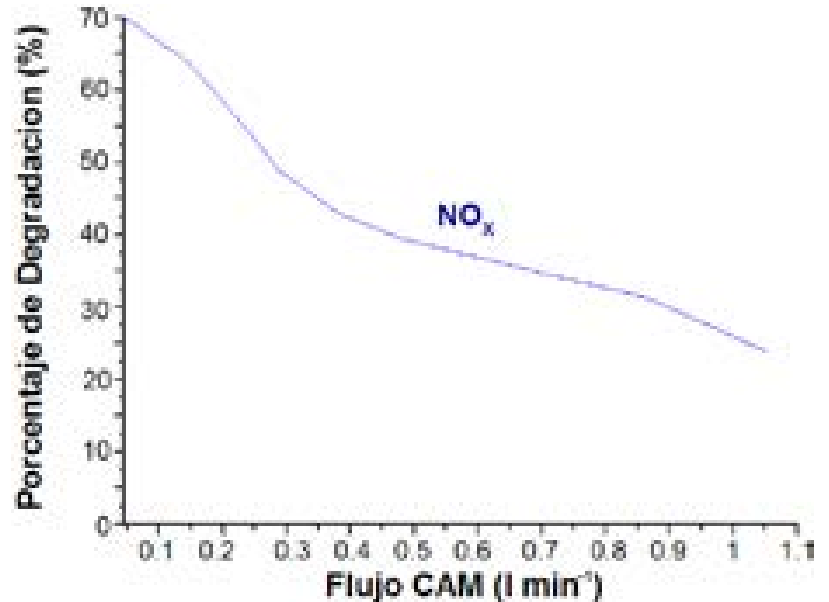

Figura 11. Resultados de las pruebas de degradación de $\mathrm{NO}_{\mathrm{x}}$

Estrada N. et al. Chemical and electrical diagnosis of two configurations of dielectric barrier discharges applied to nitric oxides degradation. Superficies y Vacio, 2011, DOI: ND.

Ferroxcube (3 de enero de 2013). U126/91/20. U. I Cores and Accessories, Datasheet [en línea]. Disponible en: http://www.ferroxcube.com/FerroxcubeCorporateReception/datasheet/ u1269120.pdf.

idman A. Plasma chemistryy, Cambridge University Press, 2008, ISBN 9780511398575.

Gottschalk C. et al. Ozonation of water and waste water, A practical guide to understanding ozone and its applications, Wiley, 2010, ISBN 9783527319626.

Iménez G. et al. Instrumenting and programming a virtual instrument for an open loop system to control gliding centrifugal thermal plasma, Gn CCE, 2012, DOI: 10.1109/ ICEEE.2012.642110

Kogelschatz U. Dielectric-barrier discharges: Their history, discharge physics, and industrial applications. Plasma Chemist

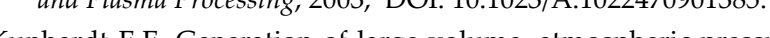
Kunhardt E.E. Generation of large-volume, atmospheric-pressuScience, 2000, DOI: 10.1109/27.842901.

Manley T.C. The electric characteristics of the ozonator discharge. Journal ffthe Electrochemical Society 1943, DOV:101149/13071556 Meichsner I. Nonthermal reactive plasmas, chapter 11, 267-298 en: Bonitz M. et al eds, Introduction to Complex Plasmas, Springer, 2010, ISBN 9783642105913. MKS (3 de enero de 2013). MKS Type 247D Four-channel readout, Instruction manual [en línee]. com/docs/R/247Dman.pdf.

MKS (3 de enero de 2013). MKS Type M100B Mass-flo controller and m10b mass-flo meter, Instruction Manual [en línea]. Disponible en: www.mksinst.com/docs/R/M100B-M10MBman.pdf. 
Mohammad, K. Switch Mode Power Supply (SMPS) Topologies (Part I), Microchip Technology, 2007, Inc., ID: AN1114. I (2013, 5 de febrero). NI 625x Specifications [en línea]. Disponble en: http://www.ni.com/pdf/manuals/375216c.pdf.

\section{Este artículo se cita:}

Citación estilo Chicago

Iménez-Aviles, Gerardo, Ricardo Valdivia-Barrientos, Arturo ColínCruz, Marquidia Pacheco-Pacheco, Joel Pacheco-Sotelo. Control de ga (3 de enero de 2013). FMA-A2100's, FMA-A2300' Massflow meters, user's manual [en línea]. Disponible en Www.omega.com/Manuals/manualpdf/M2842.pdf-

checo M.J. et al. Application of non-thermal plasma on gas

Pacheco M. et al. Removal of main exhaust gases of vehicles by a double dielectric barrier discharge, $14^{\text {th }}$ LAWPP, 2012, DOI

Pacheco MJ et al. Tratamiento de emisiones de motores diesel por plasma fuera de equilibrio. Revista Internacional de Contamin ción Ambiental, 2013, DOI: ND

Penetrante B.M. et al. Plasma-assisted ctalytic storage reductio system, Patente, 2002, US6038853 A.

Roth J.R. Industrial plasma engineering, volume 1, principles, Institute of Physics Publishing, 1995, ISBN 0750303174. in sistema de descargas de barrera dieléctrica basado en el diseño

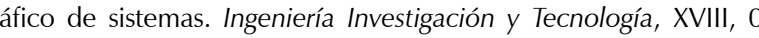

Citación estilo ISO 690

liménez-Aviles G., Valdivia-Barrientos R., Colin-Cruz A., PachecoPacheco M., Pacheco-Sotelo I. Control de un sistema de descargas de barrera dieléctrica basado en el diseño gráfico de sistemas. Ingeniería 2017: 17-29.

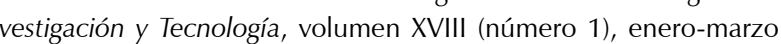

\section{Semblanzas de LOS AUtor}

Gerardo Jiménez-Aviles. Adscrito a la Facultad de Química de la Universidad Autónoma del Estado de México, casa de estudios donde obtuvo el título como ingeniero en electrónica en 2011. En 2013 concluyó sus estudios de maestría en ciencias ambienrales. Su area de interés se encuentra en las aplicaciones del GSD y del plasma con nanomateriales (ambiental y medicina). Ha nal Instruments y del Gobierno del Estado de México. Actualmente está a cargo de la Sociedad Civil Che Tuumben, de la que es socio fundador.

Ricardo Valdivia-Barrientos. Recibió el título como ingeniero electrónico por el Instituto Tecnológico de Toluca (ITT), México en 2003 donde actualmente se encuentra cursando el doctorado en ingeniería electrónica. Se ha involucrado en el desarrollo de sistedonde actualmente se encuentra cursando el doctorado en ingeniería electrónica. Se ha involucrado en el desarrollo de sistedel Instituto Nacional de Investigaciones Nucleares (ININ), Ciudad de México.

Arturo Colin-Cruz. Adscrito a la Facultad de Química de la Universidad Autónoma del Estado de México (UAEM). En 2007 obtuvo el grado de doctor en el programa de posgrado en Ingenieria de la UAEM. Actualmente es miembro del Sistema Nacional de Investigadores Nivel I, Con Linea de Generacion y Aplicación del Conocimiento "Prevención, Control y Efectos de la Contaganismos nacios

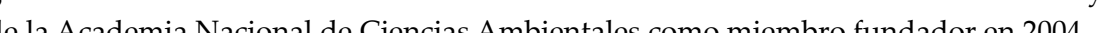

Marquidia Pacheco-Pacheco. Recibió el título en ingeniería química por el Instituto Tecnológico de Toluca, México, en 1996, y los grados de M.Sc. y Ph.D. en física e ingeniería de plasmas por la Universidad Paul Sabatier de Toulouse, Francia en 1998 y 2003, an a licacín Se nuevas tecnolóís para el tratamiento de la contaminación del aire y de no Sistema

Joel Pacheco-Sotelo. Recibió el título como ingeniero industrial en electrónica y el grado de maestro en electrónica de potencia por el Instituto de Tecnología de Chihuahua, Chihuahua, México, en 1974 y 1983, respectivamente. Asimismo el Ph.D. en el Instituto Politécnico de Toulouse, Francia en 193. Desde 1974, está adscrito al Instituto Nacional de Investigaciones Nucleares (ININ), Con

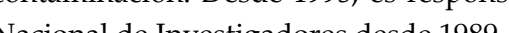

